Research Paper

\title{
Lipid Emulsion Inhibits Vasodilation Induced by a Toxic Dose of Bupivacaine via Attenuated Dephosphorylation of Myosin Phosphatase Target Subunit 1 in Isolated Rat Aorta
}

\author{
Seong-Ho Ok ${ }^{*}$, Hyo-Jin Byon $2,8^{*}$, Seong-Chun Kwon³ ${ }^{3}$ Jungchul Park ${ }^{4}$, Youngju Lee ${ }^{4}$, Yeran Hwang 4 , Jiseok \\ Baik5, Mun-Jeoung Choi ${ }^{6}$, and Ju-Tae Sohn ${ }^{1,7}, \bowtie$ \\ 1. Department of Anesthesiology and Pain Medicine, Gyeongsang National University School of Medicine and Gyeongsang National University Hospital, \\ Jinju-si, 52727, Republic of Korea; \\ 2. Department of Anesthesiology and Pain Medicine, Anesthesia and Pain Research Institute, Yonsei University College of Medicine, Seoul, Korea; \\ 3. Department of Physiology, Institute for Clinical and Translational Research, Catholic Kwandong University College of Medicine, Gangneung, 25601, Korea; \\ 4. Department of Anesthesiology and Pain Medicine, Gyeongsang National University Hospital, Jinju, 660-702, Republic of Korea; \\ 5. Department of Anesthesiology and Pain Medicine, Pusan National University Hospital, Biomed Research Institute, Pusan National University School of \\ Medicine, Busan, Republic of Korea; \\ 6. Department of Oral and Maxillofacial Surgery, Gyeongsang National University Hospital, Jinju, 660-702, Republic of Korea; \\ 7. Institute of Health Sciences, Gyeongsang National University, Jinju, Republic of Korea; \\ 8. Department of Anesthesiology and Pain Medicine, Gyeongsang National University School of Medicine, Jinju-si, 52727, Republic of Korea
}

*These authors contributed equally to this study as co-first authors.

$\triangle$ Corresponding author: Ju-Tae Sohn, Department of Anesthesiology and Pain Medicine, Gyeongsang National University Hospital, Jinju, 660-702, Republic of Korea; Tel.: +82-55-750-8586; Fax: +82-55-750-8142; E-mail: jtsohn@nongae.gsnu.ac.kr

(C) 2015 Ivyspring International Publisher. Reproduction is permitted for personal, noncommercial use, provided that the article is in whole, unmodified, and properly cited. See http://ivyspring.com/terms for terms and conditions.

Received: 2015.07.21; Accepted: 2015.10.10; Published: 2015.11.05

\begin{abstract}
Lipid emulsions are widely used for the treatment of systemic toxicity that arises from local anesthetics. The goal of this in vitro study was to examine the cellular mechanism associated with the lipid emulsion-mediated attenuation of vasodilation induced by a toxic dose of bupivacaine in isolated endothelium-denuded rat aorta. The effects of lipid emulsion on vasodilation induced by bupivacaine, mepivacaine, and verapamil were assessed in isolated aorta precontracted with phenylephrine, the Rho kinase stimulant $\mathrm{NaF}$, and the protein kinase $\mathrm{C}$ activator phorbol 12,13-dibutyrate (PDBu). The effects of Rho kinase inhibitor Y-27632 on contraction induced by phenylephrine or $\mathrm{NaF}$ were assessed. The effects of bupivacaine on intracellular calcium concentrations $\left(\left[\mathrm{Ca}^{2+}\right]_{\mathrm{i}}\right)$ and tension induced by $\mathrm{NaF}$ were simultaneously measured. The effects of bupivacaine alone and lipid emulsion plus bupivacaine on myosin phosphatase target subunit 1 (MYPTI) phosphorylation induced by $\mathrm{NaF}$ were examined in rat aortic vascular smooth muscle cells. In precontracted aorta, the lipid emulsion attenuated bupivacaine-induced vasodilation but had no effect on mepivacaine-induced vasodilation. Y-27632 attenuated contraction induced by either phenylephrine or $\mathrm{NaF}$. The lipid emulsion attenuated verapamil-induced vasodilation. Compared with phenylephrine-induced precontracted aorta, bupivacaine-induced vasodilation was slightly attenuated in NaF-induced precontracted aorta. The magnitude of the bupivacaine-induced vasodilation was higher than that of a bupivacaine-induced decrease in $\left[\mathrm{Ca}^{2+}\right]_{i}$. Bupivacaine attenuated NaF-induced MYPT1 phosphorylation, whereas lipid emulsion pretreatment attenuated the bupivacaine-induced inhibition of MYPTI phosphorylation induced by NaF. Taken together, these results suggest that lipid emulsions attenuate bupivacaine-induced vasodilation via the attenuation of inhibition of MYPTI phosphorylation evoked by $\mathrm{NaF}$.
\end{abstract}

Key words: aorta, bupivacaine, lipid emulsion, phenylephrine, vasodilation, myosin phosphatase target subunit 1 


\section{Introduction}

Lipid emulsions are effective treatments for cardiac arrest caused by toxic doses of bupivacaine and levobupivacaine [1,2]. In addition, lipid emulsions such as SMOFlipid ${ }^{\circledR}$, Intralipid ${ }^{\circledR}$, and Lipofundin ${ }^{\circledR}$ MCT/LCT (medium-chain triglycride and long-chain triglyceride) reverse severe vasodilation induced by toxic doses of levobupivacaine and bupivacaine, suggesting that lipid emulsions are involved in the recovery of vascular tone from vascular collapse caused by toxic doses of bupivacaine and levobupivacaine via the sequestration of local anesthetics in a lipid solubility dependent manner [3-5]. The proposed mechanisms of lipid emulsion therapy include lipid sink (sequestration of local anesthetic), fatty acid supply, reversal of sodium channel blockade, cytoprotection, calcium entry and pharmacokinetic effect [2].

Low doses of levobupivacaine produce vasoconstriction at the resting tension, whereas high doses of levobupivacaine cause vasodilation, which appears to be associated with vascular collapse evoked by such high doses of levobupivacaine [6-8]. In addition, a toxic dose of bupivacaine induces vasodilation that appears to be partially associated with decreased calcium sensitization in isolated endothelium-denuded rat aorta precontracted with phenylephrine [9]. Agonists, including phenylephrine, activate Rho kinase and protein kinase $\mathrm{C}$ (PKC), which leads to contraction via increased calcium sensitization [10]. Rho kinase and PKC phosphorylate myosin phosphatase target subunit 1 (MYPT1) and phosphorylation-dependent inhibitory protein of myosin phosphatase, respectively, leading to increased calcium sensitization via the inhibition of myosin light chain phosphatase (MLCP) [10]. Because the phosphorylation of MYPT1, which is a signaling molecule downstream of Rho kinase that participates in vascular smooth muscle contraction, is involved in calcium sensitization, whereas bupivacaine-induced vasodilation causes decreased calcium sensitization, we tested the hypothesis that lipid emulsion attenuated bupivacaine-induced vasodilation via the modulation of MYPT1 phosphorylation [3-5,9,10]. The goal of this in vitro study was to investigate the cellular mechanism responsible for the lipid emulsion-mediated attenuation of severe vasodilation induced by a toxic dose of bupivacaine, focusing on MYPT1 phosphorylation. The severe vasodilation was induced by a toxic dose of bupivacaine in isolated endothelium-denuded rat aortas precontracted with vasoconstrictors such as phenylephrine or the Rho kinase activator, $\mathrm{NaF}$.

\section{Materials and methods}

All experimental procedures and protocols were approved by the Institutional Animal Care and Use Committee at Gyeongsang National University. All experimental procedures were performed in accordance with the Guide for the Care and Use of Laboratory Animals prepared by the Institute for Laboratory Animal Research.

\section{Preparation of aortic rings for tension meas- urements}

Preparation of aortic rings for tension measurements was performed as previously described [4]. Male Sprague-Dawley rats weighing 250-300 g were anesthetized by intramuscular injection of zoletil 50 (15 mg/kg, Virbac Laboratories, Carros, France). The descending thoracic aorta was dissected free of surrounding connective tissues and fat under microscopic guidance in a Krebs solution bath consisting of $118 \mathrm{mM} \mathrm{NaCl}, 4.7 \mathrm{mM} \mathrm{KCl}, 1.2 \mathrm{mM} \mathrm{MgSO}_{4}, 1.2 \mathrm{mM}$ $\mathrm{KH}_{2} \mathrm{PO}_{4}, 2.4 \mathrm{mM} \mathrm{CaCl}_{2}, 25 \mathrm{mM} \mathrm{NaHCO} 3$, and $11 \mathrm{mM}$ glucose. The endothelium was removed from all aortic rings by inserting a 25-gauge needle into the lumen of the rings and gently rubbing the ring for a few seconds. The aorta was suspended on a Grass isometric transducer (FT-03, Grass Instrument, Quincy, MA, USA) under a $3.0-\mathrm{g}$ resting tension in a bath containing $10 \mathrm{~mL}$ Krebs solution at $37^{\circ} \mathrm{C}$ and aerated continuously with $95 \% \mathrm{O}_{2}$ and $5 \% \mathrm{CO}_{2}$ to maintain the $\mathrm{pH}$ between 7.35-7.45. The rings were equilibrated at a 3.0-g resting tension for $120 \mathrm{~min}$, and the bath solution was changed every $30 \mathrm{~min}$. After contractions induced by $10^{-8} \mathrm{M}$ phenylephrine had stabilized, endothelial denudation of the aortic rings was verified by observation of less than $15 \%$ relaxation in response to acetylcholine $\left(10^{-5} \mathrm{M}\right)$. After washing out the phenylephrine from the organ bath and allowing a return of the isometric tension to baseline, the main experiments were performed according to the experimental protocols described below. Each ring was used for only one concentration-response curve induced by bupivacaine, mepivacaine, verapamil, phenylephrine, or $\mathrm{NaF}$. Because increase in left ventricular systolic pressure by lipid emulsion seems to be associated with the blockade of nitric oxide in an in vivo state with endothelium-intact aorta, the Krebs solution contained the nitric oxide synthase inhibitor $\mathrm{NW}^{\mathrm{W}}$-nitro-L-arginine methyl ester (L-NAME, $10^{-4} \mathrm{M}$ ) to prevent the release of endogenous nitric oxide from the residual endothelium [11].

\section{Experimental protocols}

The first series of experiments was designed to examine the effects of lipid emulsions (Intralipid ${ }^{\circledR}$, 
0.35 and $0.8 \%$ ) on the vasodilation induced by bupivacaine or mepivacaine in endothelium-denuded aorta precontracted with phenylephrine or $\mathrm{NaF}$. Lipid emulsions $(0.35$ and $0.8 \%)$ were added into the organ bath for $20 \mathrm{~min}$ before the addition of phenylephrine $\left(10^{-6} \mathrm{M}\right)$ or $\mathrm{NaF}\left(8 \times 10^{-3} \mathrm{M}\right)$. After the phenylephrineor NaF-induced contraction had stabilized, incremental concentrations of bupivacaine $\left(10^{-6}\right.$ to $\left.10^{-3} \mathrm{M}\right)$ or mepivacaine $\left(10^{-5}\right.$ to $\left.10^{-2} \mathrm{M}\right)$ were added to the organ bath to generate concentration-response curves [12]. In addition, we examined the effects of the lipid emulsion (Intralipid ${ }^{\circledR}, 0.35$ and $0.8 \%$ ) alone on contraction induced by phenylephrine $\left(10^{-6} \mathrm{M}\right)$ or $\mathrm{NaF}(8$ $\left.\times 10^{-3} \mathrm{M}\right)$ in isolated endothelium-denuded aorta. After the contraction induced by $\mathrm{NaF}$ or phenylephrine stabilized in isolated endothelium-denuded aorta, the lipid emulsion ( 0.35 and $0.8 \%$ ) was added to the organ bath to examine changes in tension induced by $\mathrm{NaF}$ or phenylephrine.

The second series of experiments was designed to examine the effect of Rho kinase inhibitor Y-27632 on the contraction induced by phenylephrine $\left(10^{-6} \mathrm{M}\right)$ or $\mathrm{NaF}\left(8 \times 10^{-3} \mathrm{M}\right)$ in endothelium-denuded aorta. After phenylephrine- or NaF-induced contraction had stabilized, one of three different concentrations of Y-27632 $\left(10^{-6}, 3 \times 10^{-6}\right.$, and $\left.10^{-5} \mathrm{M}\right)$ was directly added to the organ bath, and the tension was continuously measured for $60 \mathrm{~min}$.

The third series of experiments was designed to examine the effects of various vasoconstrictors on bupivacaine-induced vasodilation in endothelium-denuded aorta. After contraction induced by phenylephrine $\left(10^{-6} \mathrm{M}\right), \mathrm{NaF}\left(8 \times 10^{-3} \mathrm{M}\right)$, and PKC activator phorbol $12,13-$ dibutyrate $\left(\mathrm{PDBu}, 10^{-6} \mathrm{M}\right)$ had stabilized, incremental concentrations $\left(10^{-6}\right.$ to $\left.10^{-3} \mathrm{M}\right)$ of bupivacaine were added to the organ bath to compare the patterns of bupivacaine-induced vasodilation in aorta precontracted with the various vasoconstrictors.

Finally, we investigated the effects of the lipid emulsion (Intralipid ${ }^{\circledR}, 0.25 \%$ ) on vasodilation induced by verapamil (a highly lipid soluble calcium channel blocker) in aorta precontracted with phenylephrine to examine whether the lipid emulsion-mediated attenuation of bupivacaine-induced vasodilation is specific or nonspecific [13]. The lipid emulsion was added to the organ bath for $20 \mathrm{~min}$ before the addition of phenylephrine $\left(10^{-7} \mathrm{M}\right)$. After the phenylephrine-induced contraction had reached a plateau, incremental concentrations of verapamil $\left(10^{-9}\right.$ to $\left.10^{-5} \mathrm{M}\right)$ were added to the organ bath to generate verapamil dose-response curves.

\section{Fura-2 loading and simultaneous measure- ments of intracellular calcium concentration $\left(\left[\mathrm{Ca}^{2+}\right]_{i}\right)$ and tension}

$\left[\mathrm{Ca}^{2+}\right]_{\mathrm{i}}$ and tension were simultaneously measured as described previously [9,14]. Muscle strips were exposed to the acetoxymethyl ester of fura-2 (fura-2/AM, $10 \mu \mathrm{M}$ ) in the presence of $0.02 \%$ cremophor EL for 5-6 h at room temperature. After loading, a muscle strip was washed with Krebs solution at $37^{\circ} \mathrm{C}$ for $20 \mathrm{~min}$ to remove any uncleaved fura-2/ AM and held horizontally in a temperature controlled, 7-ml organ bath. One end of the muscle strip was connected to a force-displacement transducer (MLT050, AD Instruments, Colorado Springs, CO, USA) to monitor muscle contraction. The muscle strip was illuminated alternately $(48 \mathrm{~Hz})$ at two excitation wavelengths (340 and $380 \mathrm{~nm}$ ). The intensity of the 500-nm fluorescence (F340 and F380) was measured with a fluorometer (CAF-110, Jasco, Tokyo, Japan). The ratio of F340 to F380 (F340/F380) was calculated as an indicator of $\left[\mathrm{Ca}^{2+}\right]_{i}$. Therefore, the ratio (F340/F380) and tension obtained from $8 \times 10^{-3} \mathrm{M}$ $\mathrm{NaF}$-stimulated aortic strips were taken as 100 and $100 \%$, respectively. Isometric contractions and the ratio of F340/F380 were recorded with a PowerLab/400 using the chart program (AD instruments). Muscle strips were placed under an initial 3.0-g resting tension. All strips that came from the same animal were used in different experimental protocols. When both $\left[\mathrm{Ca}^{2+}\right]_{\mathrm{i}}$ and contraction induced by $8 \times 10^{-3} \mathrm{M} \mathrm{NaF}$ reached steady-state levels, incremental concentrations of bupivacaine $\left(10^{-6}\right.$ to $\left.10^{-3} \mathrm{M}\right)$ were added cumulatively.

\section{Cell culture}

Vascular smooth muscle cells (VSMCs) were isolated from thoracic aorta of male rats by enzymatic dissociation and grown in Dulbecco's modified Eagle's medium supplemented with $10 \%$ heatinactivated fetal bovine serum, $2 \mathrm{mM}$ L-glutamine, 100 $\mathrm{U} / \mathrm{ml}$ penicillin, and $100 \mu \mathrm{g} / \mathrm{ml}$ streptomycin as described previously [14]. Cells were subcultured twice per week by harvesting with trypsin/ethylenediaminetetraacetic acid and seeding into flasks at a density of $7.5 \times 10^{5} / \mathrm{mm}^{2}$. For experiments, cells between passage numbers 2 and 10 were seeded into dishes (107/100-mm dish), fed every other day, and used at confluence (6-7 days). Cells were deprived of serum overnight prior to treatment.

\section{Western blot analysis}

Western blot analysis was performed as described previously [14]. Cells were lysed in PRO-PREP protein extract solution (iNtRON Bio- 
technology, Houston, TX, USA) to obtain total cell lysates, and the lysates were centrifuged at $100,000 \times g$ for $20 \mathrm{~min}$ at $4^{\circ} \mathrm{C}$. Protein concentrations were determined using the Bradford method. For preparation of sample loading, equal volumes of $2 \times$ sodium dodecyl sulfate sample buffer $(0.1 \mathrm{~mol} / \mathrm{L}$ Tris-HCI, $20 \%$ glycerol, $4 \%$ sodium dodecyl sulfate, and $0.01 \%$ bromophenol blue) and supernatant fractions from the lysates were mixed. Proteins $(60 \mu \mathrm{g})$ were separated by $10 \%$ sodium dodecyl sulfate-polyacrylamide gel electrophoresis for $90 \mathrm{~min}$ at $110 \mathrm{~V}$. The separated proteins were transferred onto polyvinylidene difluoride membranes for $2 \mathrm{~h}$ at $20 \mathrm{~mA}$ using SD Semi-dry Transfer Cells (Bio-Rad Laboratories, Hercules, CA, USA). After blocking the membranes using $5 \%$ nonfat milk in Tris-buffed saline ( $\mathrm{pH} 7.0)$, the membranes were incubated overnight at $4^{\circ} \mathrm{C}$ with primary antibodies (anti-phospho-MYPT1 and anti-MYPT1 antibodies) at a dilution of $1: 500$ in 5\% skim milk in Tris-buffed saline containing Tween-20. Bound antibody was detected with horseradish peroxidase-conjugated anti-mouse IgM. Membranes were washed and developed using the Western Blotting Luminol Reagent system (iNtRON Biotechnology) and autoradiography.

\section{Materials}

All drugs were of the highest purity available commercially. Phenylephrine, NaF, PDBu, acetylcholine, and L-NAME were obtained from Sigma Aldrich (St. Louis, MO, USA). Y-27632 was obtained from Calbiochem (La Jolla, CA, USA). Fura-2/AM was obtained from Molecular Probes (Eugen, OR, USA). Anti-phospho-MYPT1 and anti-MYPT1 antibodies were obtained from Cell Signaling Technology (Beverly, MA, USA). Intralipid ${ }^{\circledR} 20 \%$ was obtained from Fresenius Kabi Korea (Seoul, Korea). Bupivacaine was obtained from Reyon Pharmaceutical Co., Ltd. (Seoul, Korea). Mepivacaine was donated by Hana Pharmaceutical Co., Ltd. (Gyeonggi-do, Korea). All concentrations except the lipid emulsions were expressed as the final molar concentration in the organ bath. PDBu and fura-2/AM were dissolved in dimethyl sulfoxide (final organ bath concentration: $0.01 \%$ ). Unless stated otherwise, all drugs were dissolved in distilled water.

\section{Data analysis}

The values are shown as the mean \pm SD. Vasodilation induced by bupivacaine, mepivacaine, Y-27632, and verapamil is expressed as the percentage of the maximum contraction induced by phenylephrine, NaF, and PDBu. The effect of the lipid emulsion on vasodilation induced by bupivacaine or mepivacaine in endothelium-denuded aorta precontracted with phenylephrine or $\mathrm{NaF}$ was analyzed us- ing two-way repeated measures analysis of variance (ANOVA) followed by Bonferroni's post-test (Prism 5.0, GraphPad Software, San Diego, CA, USA). The effect of the lipid emulsion alone on contraction induced by phenylephrine or $\mathrm{NaF}$ in endothelium-denuded aorta was analyzed using two-way repeated measures ANOVA followed by Bonferroni's post-test. The logarithm of drug concentration $\left(\mathrm{ED}_{50}\right)$ producing $50 \%$ of the maximal relaxation induced by verapamil was calculated with nonlinear regression analysis by fitting the concentration-response relationship for verapamil to a sigmoidal curve using commercially available software (Prism 5.0, GraphPad Software). Statistical analysis for the comparison of $\mathrm{ED}_{50}$ and maximal relaxation between the control and lipid emulsion-treated groups during verapamil-induced relaxation was performed using Mann-Whitney $U$ test. The effects of vasoconstrictors on bupivacaine-induced vasodilation and of Y-27632 on contraction induced by phenylephrine and $\mathrm{NaF}$ were analyzed using two-way repeated measures ANOVA followed by Bonferroni's post-test. Analyses regarding effects of bupivacaine alone and lipid emulsion plus bupivacaine on the NaF-induced MYPT1 phosphorylation were performed using one-way ANOVA followed by Bonferroni's post-test. The effect of bupivacaine on the tension and $\left[\mathrm{Ca}^{2+}\right]_{\mathrm{i}}$ induced by $\mathrm{NaF}$ was analyzed using two-way repeated measures ANOVA followed by Bonferroni's post-test. Scanning densitometry was performed using an Image Master VSD (Pharmacia Biotech, San Francisco, CA, USA). $P$ values less than 0.05 were statistically significant.

\section{Results}

The lipid emulsion (0.35 and $0.8 \%)$ attenuated bupivacaine-induced vasodilation in endothelium-denuded aorta precontracted with phenylephrine (Fig. 1A; $P<0.05$ versus control at $3 \times 10^{-4} \mathrm{M}$ bupivacaine). However, the lipid emulsion had no effect on mepivacaine-induced vasodilation under similar conditions (Fig. 2A). In addition, in endothelium-denuded aorta precontracted with $\mathrm{NaF}$, the lipid emulsion (0.35 and $0.8 \%)$ attenuated bupivacaine-induced vasodilation in a concentration-dependent manner (Fig. 1B; $P<0.05$ versus control at $10^{-4}$ and $3 \times 10^{-4} \mathrm{M}$ bupivacaine), whereas the lipid emulsion had no effect on mepivacaine-induced vasodilation (Fig. 2B). The lipid emulsion (0.35 and $0.8 \%$ ) had no significant effect on aorta precontracted with $\mathrm{NaF}\left(8 \times 10^{-3} \mathrm{M}\right)$ or phenylephrine $\left(10^{-6} \mathrm{M}\right)$ compared with time-matched controls (data not shown). All doses (10-6 to $\left.10^{-5} \mathrm{M}\right)$ of Y-27632 attenuated phenylephrine-induced contraction in endothe- 
lium-denuded aorta (Fig. 3A; $P<0.01$ versus control at 20 to $60 \mathrm{~min}$ ) as well as NaF-induced contraction (Fig. 3B; $P<0.001$ versus control at 10 to $60 \mathrm{~min}$ ). Bupivacaine-induced vasodilation was slightly attenuated in NaF-induced precontracted aorta compared with phenylephrine-induced precontracted aorta (Fig. 4; $P<0.05$ versus phenylephrine at $3 \times 10^{-5}$ and $3 \times 10^{-4}$ $\mathrm{M}$ bupivacaine), whereas bupivacaine-induced vaso- dilation was greatly attenuated in PDBu-induced precontracted aorta compared with phenylephrine-induced precontracted aorta (Fig. 4; $P<$ 0.05 versus phenylephrine at $3 \times 10^{-5}$ to $10^{-3} \mathrm{M}$ bupivacaine). The lipid emulsion $(0.25 \%)$ attenuated verapamil-induced vasodilation in aorta precontracted with phenylephrine (Fig. 5; $\mathrm{ED}_{50}$ : $P<0.05$ versus control).
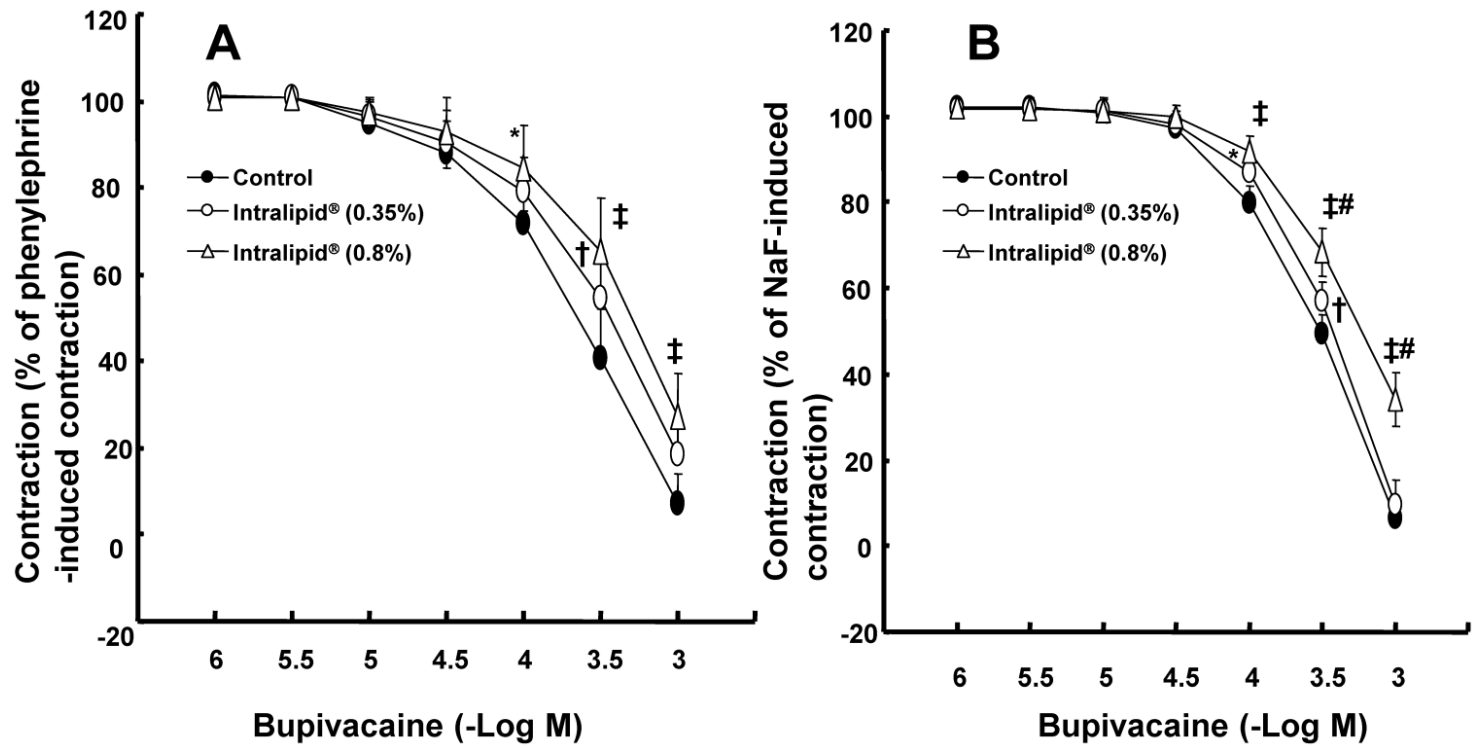

Figure 1. Effects of lipid emulsions (Intralipid ${ }^{\circledR}, 0.35$ and $0.8 \%$ ) on bupivacaine-induced vasodilation in isolated endothelium-denuded aorta precontracted with $10-6$ M phenylephrine $(A)$ or $8 \times 10^{-3} \mathrm{M} \mathrm{NaF}(B)$. All values are shown as the mean $\pm S D(n=6)$ and expressed as the percentage of the maximal contraction induced by phenylephrine or $\mathrm{NaF} . \mathrm{N}$ indicates the number of rats from which descending thoracic aortic rings were derived. $A: * P<0.05, \dagger P<0.01$, and $\ddagger P<0.001$ versus control. $B$ : $* P<0.05$, $\nmid P<0.01$, and $\ddagger P<0.001$ versus control. \#P<0.001 versus $0.35 \%$ Intralipid ${ }^{\circledR}$.
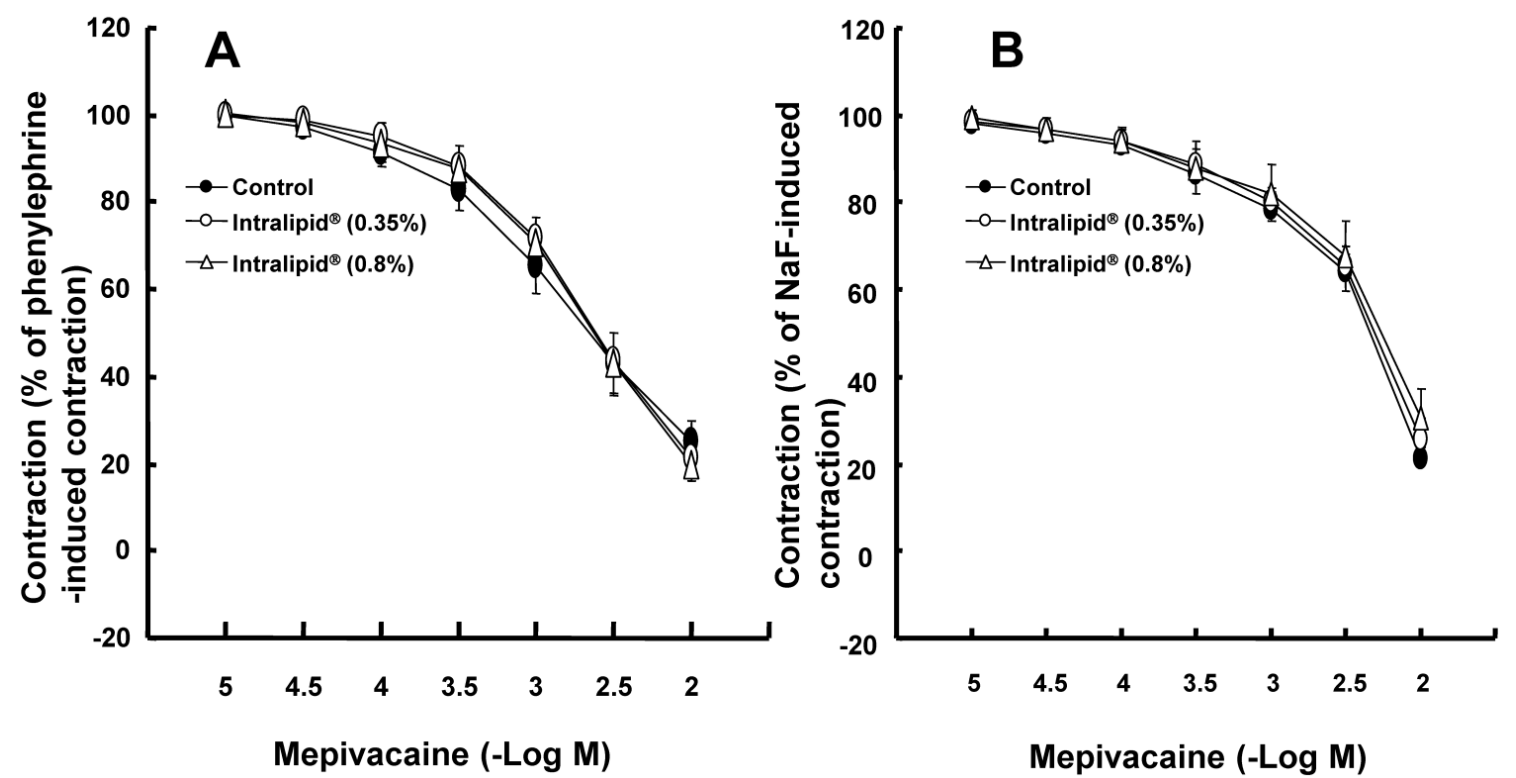

Figure 2. Effects of lipid emulsions (Intralipid ${ }^{\circledR}, 0.35$ and $0.8 \%$ ) on mepivacaine-induced vasodilation in isolated endothelium-denuded aorta precontracted with $10-6$ M phenylephrine $(A)$ or $8 \times 10^{-3} \mathrm{M} \mathrm{NaF}(\mathrm{B})$. All values are shown as the mean $\pm \mathrm{SD}(\mathrm{n}=5)$ and expressed as the percentage of the maximal contraction induced by phenylephrine or $\mathrm{NaF} . \mathrm{N}$ indicates the number of rats from which descending thoracic aortic rings were derived. 

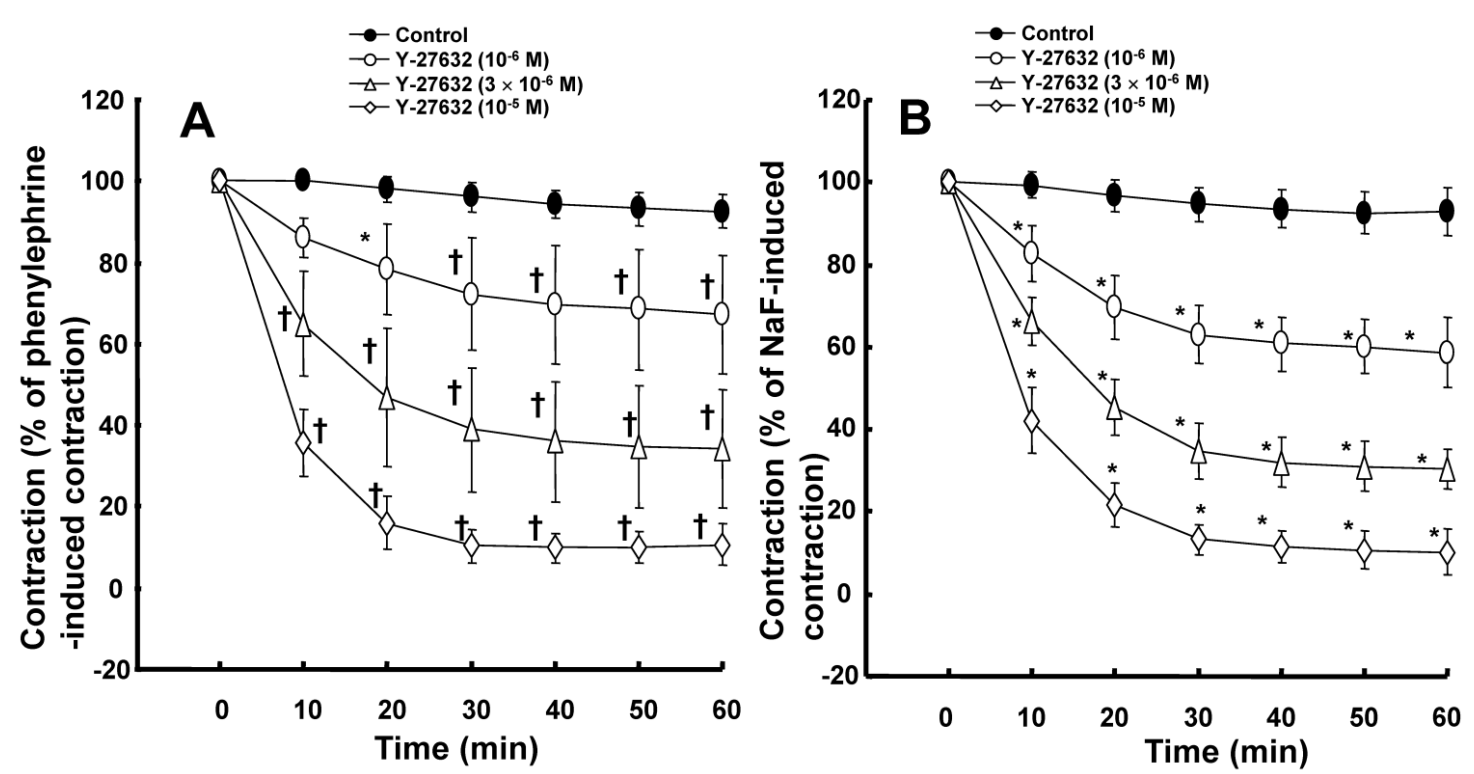

Figure 3. A: Effects of $Y-27632$ on phenylephrine (10-6 M)-induced contraction in isolated endothelium-denuded aorta. All values are shown as the mean \pm SD ( $=7$ ) and expressed as the percentage of the maximal contraction induced by phenylephrine. $N$ indicates the number of rats from which descending thoracic aortic rings were derived. $* P$ $<0.01$ and $+P<0.001$ versus control. B: Effects of $\mathrm{Y}-27632$ on $\mathrm{NaF}\left(8 \times 10^{-3} \mathrm{M}\right)$-induced contraction in isolated endothelium-denuded aorta. All values are shown as the mean $\pm S D(n=6)$ and expressed as the percentage of the maximal contraction induced by NaF. $N$ indicates the number of descending thoracic aortic rings. $* P<0.001$ versus control.

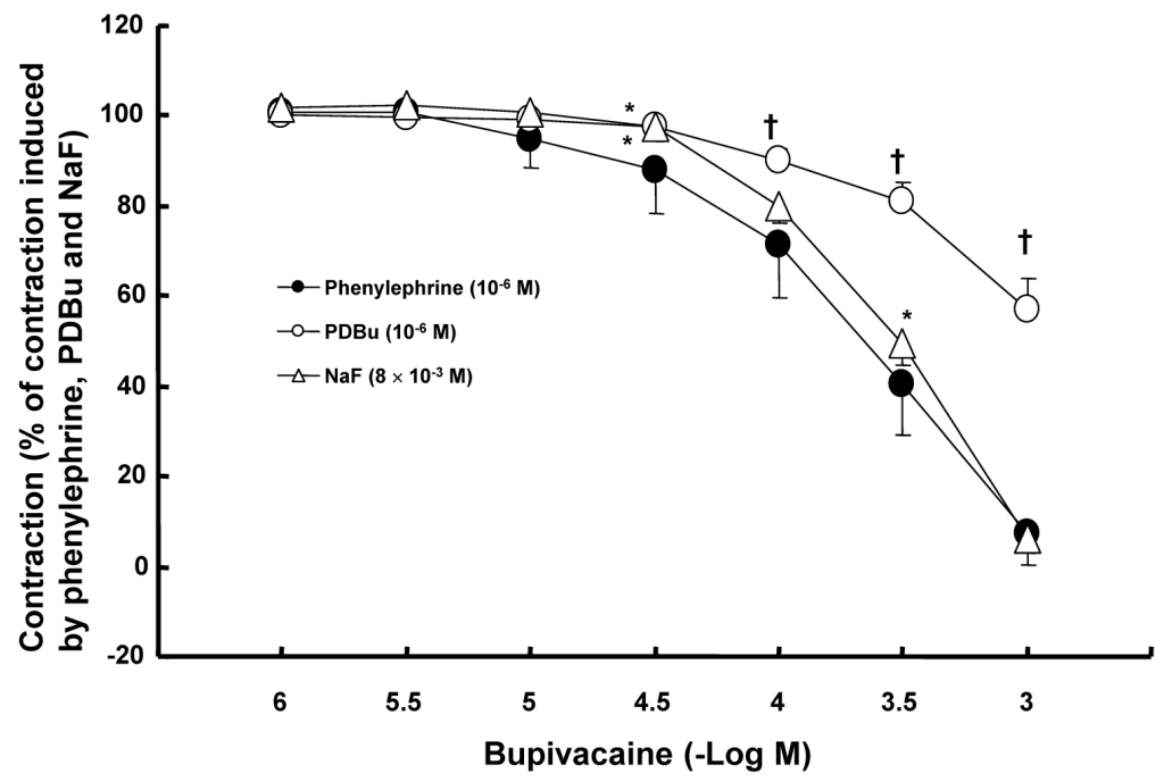

Figure 4. Bupivacaine-induced vasodilation in isolated endothelium-denuded aorta precontracted with 10-6 M phenylephrine, $8 \times 10^{-3} \mathrm{M}$ NaF, and phorbol 12,13-dibutyrate $(\mathrm{PDBu}, 10-6 \mathrm{M})$. All values are shown as the mean $\pm \mathrm{SD}(\mathrm{n}=6)$ and expressed as the percentage of the maximal contraction induced by phenylephrine, $\mathrm{NaF}$, and PDBu. $\mathrm{N}$ indicates the number of rats from which descending thoracic aortic rings were derived. $* P<0.05$ and $\dagger P<0.001$ versus phenylephrine.

The magnitude of bupivacaine-induced vasodilation was higher than that of the bupivacaine-induced decrease in $\left[\mathrm{Ca}^{2+}\right]_{\mathrm{i}}$ in aorta precontracted with $8 \times 10^{-3} \mathrm{M} \mathrm{NaF}$ (Fig. 6; $P<0.001$ versus F340/F380 at $3 \times 10^{-5}$ to $10^{-3} \mathrm{M}$ bupivacaine).

$\mathrm{NaF}\left(8 \times 10^{-3} \mathrm{M}\right)$ induced MYPT1 phosphorylation in rat aortic VSMCs (Fig. 7; $P<0.001$ versus control). Bupivacaine $\left(3 \times 10^{-4} \mathrm{M}\right)$ attenuated NaF-induced MYPT1 phosphorylation (Fig. 7; $P<0.01$ versus $\mathrm{NaF}$ alone), whereas the lipid emulsion $(0.8 \%)$ inhibited the bupivacaine-induced attenuation of
MYPT1 phosphorylation evoked by NaF (Fig. 7; $P<$ 0.05 versus combined treatment with bupivacaine and $\mathrm{NaF})$.

\section{Discussion}

This is first study to suggest that lipid emulsions attenuate vasodilation induced by a toxic dose of bupivacaine via the attenuation of the inhibition of MYPT1 phosphorylation induced by bupivacaine. The major findings of this in vitro study are as follows: 1 ) The lipid emulsion attenuates vasodilation induced 
by a toxic dose of bupivacaine in isolated aorta precontracted with phenylephrine or $\mathrm{NaF}$, but had no effect on vasodilation induced by a toxic dose of mepivacaine; 2) the magnitude of bupivacaine-induced vasodilation slightly differed in isolated aorta precontracted with phenylephrine or $\mathrm{NaF}$;
3) the magnitude of bupivacaine-induced vasodilation was higher than that of the bupivacaine-induced decrease in $\left[\mathrm{Ca}^{2+}\right]_{\mathrm{i}}$ in NaF-stimulated aortic strips; and 4) the lipid emulsion inhibited the bupivacaine-induced attenuation of MYPT1 phosphorylation induced by $\mathrm{NaF}$.

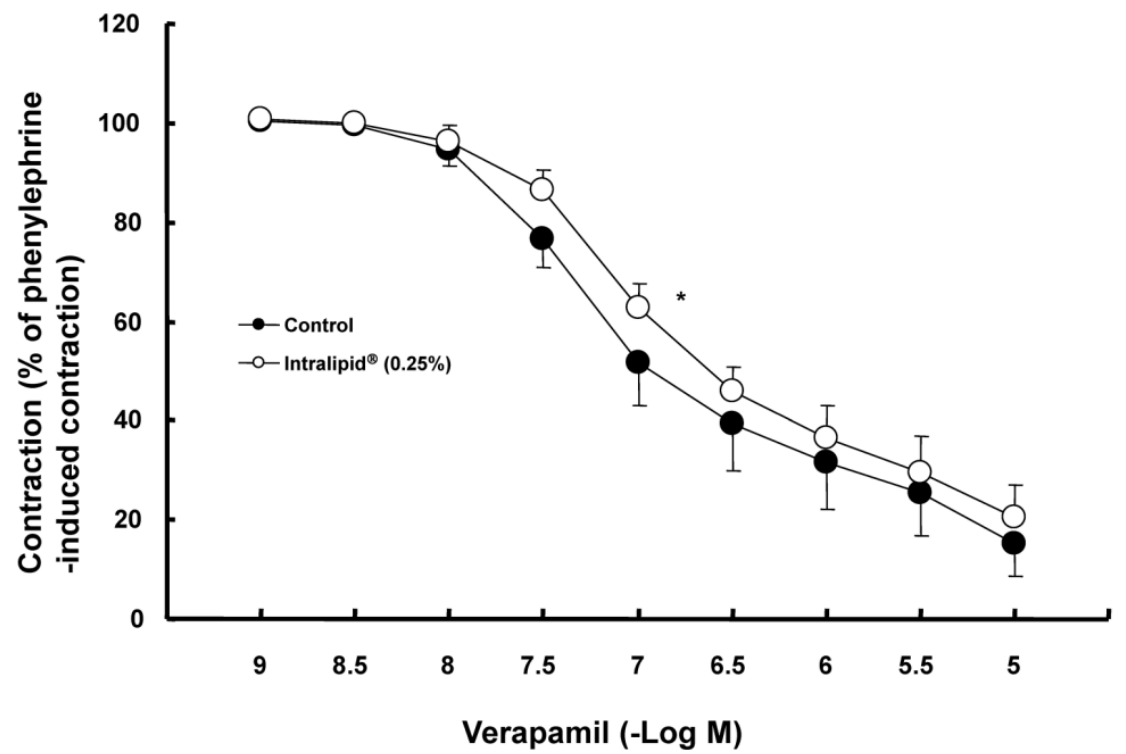

Figure 5. Effects of the lipid emulsion (Intralipid ${ }^{\circledR}, 0.25 \%$ ) on verapamil-induced vasodilation in isolated endothelium-denuded aorta precontracted with $10^{-7} \mathrm{M}$ phenylephrine. All values are shown as the mean $\pm S D(n=5)$ and expressed as the percentage of the maximal contraction induced by phenylephrine. $N$ indicates the number of rats from which descending thoracic aortic rings were derived. $\mathrm{ED}_{50}: * P<0.05$ versus control.
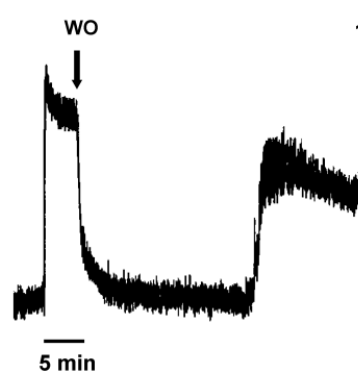

$\left|3 \times 10^{-6}\right| 3 \times 10^{-5} \mid$

$\mid \begin{gathered}0^{-4} \\ \mid 1^{-4}\end{gathered}$

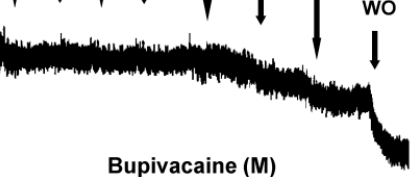

A

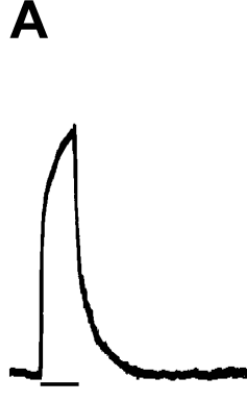

$60 \mathrm{mM} \mathrm{KCl}$

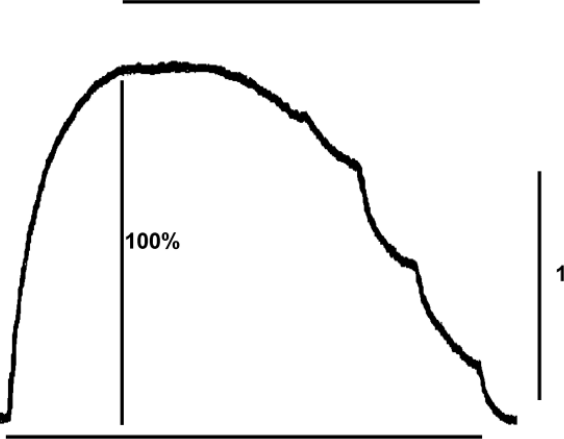

$\operatorname{NaF}\left(8 \times 10^{-3} \mathrm{M}\right)$

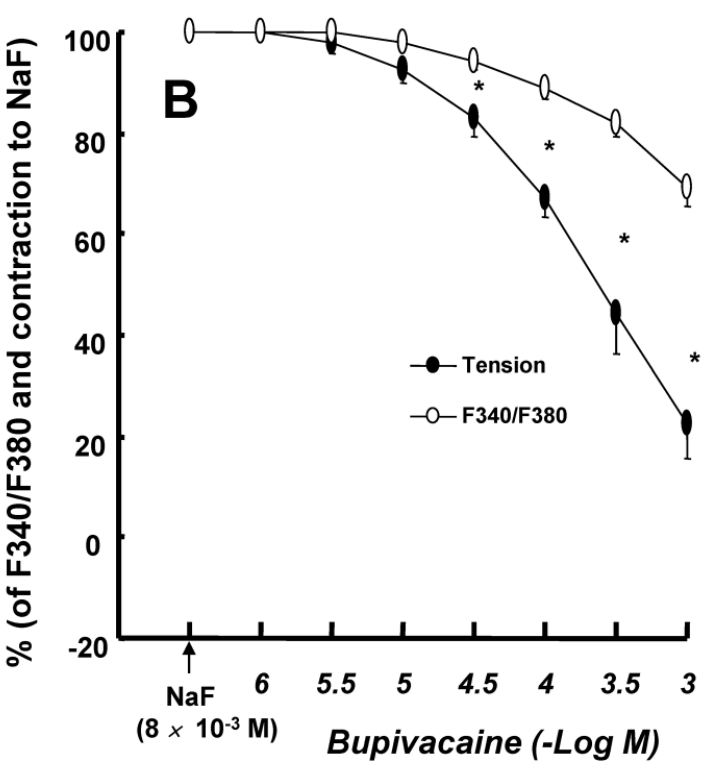

Figure 6. A: Effects of bupivacaine on $\mathrm{NaF}\left(8 \times 10^{-3} \mathrm{M}\right)$-stimulated intracellular calcium concentration ([Ca $\left.\left.{ }^{2+}\right]_{i}\right)$ (upper trace) and muscle tension (lower trace) in endothelium-denuded rat thoracic aortic strips. The $\left[\mathrm{Ca}^{2+}\right]_{i}$ of fura-2-loaded aortic strips was detected using a fluorometer and expressed as the ratio $\mathrm{F} 340 / \mathrm{F} 380$. The value $100 \%$ represents the $\mathrm{NaF}\left(8 \times 10^{-3} \mathrm{M}\right)$-induced increases in both $\left[\mathrm{Ca}^{2+}\right]_{i}$ and muscle tension before the cumulative addition of bupivacaine. When $\left[\mathrm{Ca}{ }^{2+}\right]_{i}$ and muscle tension induced by $8 \times 10^{-3} \mathrm{M} \mathrm{NaF}$ had reached steady-state levels, incremental concentrations of bupivacaine (10-6 to 10-3 M) were cumulatively added. WO: washout. B: Cumulative concentration-response curve for bupivacaine in $\mathrm{NaF}\left(8 \times 10^{-3} \mathrm{M}\right)$-stimulated endothelium-denuded rat thoracic aortic strips. Incremental concentrations (10-6 to $\left.10^{-3} \mathrm{M}\right)$ of bupivacaine were cumulatively added during the sustained increases in both $\left[\mathrm{Ca}^{2+}\right]_{i}$ and tension induced by $\mathrm{NaF}\left(8 \times 10^{-3} \mathrm{M}\right)$. The value $100 \%$ represents the NaF $\left(8 \times 10^{-3} \mathrm{M}\right)$-induced increase in both $\left[\mathrm{Ca}^{2+}\right]_{i}$ and muscle tension before the cumulative addition of bupivacaine. Each point represents the mean of five experiments, and SD is shown by vertical bars. $* P<0.001$ versus F340/F380. 

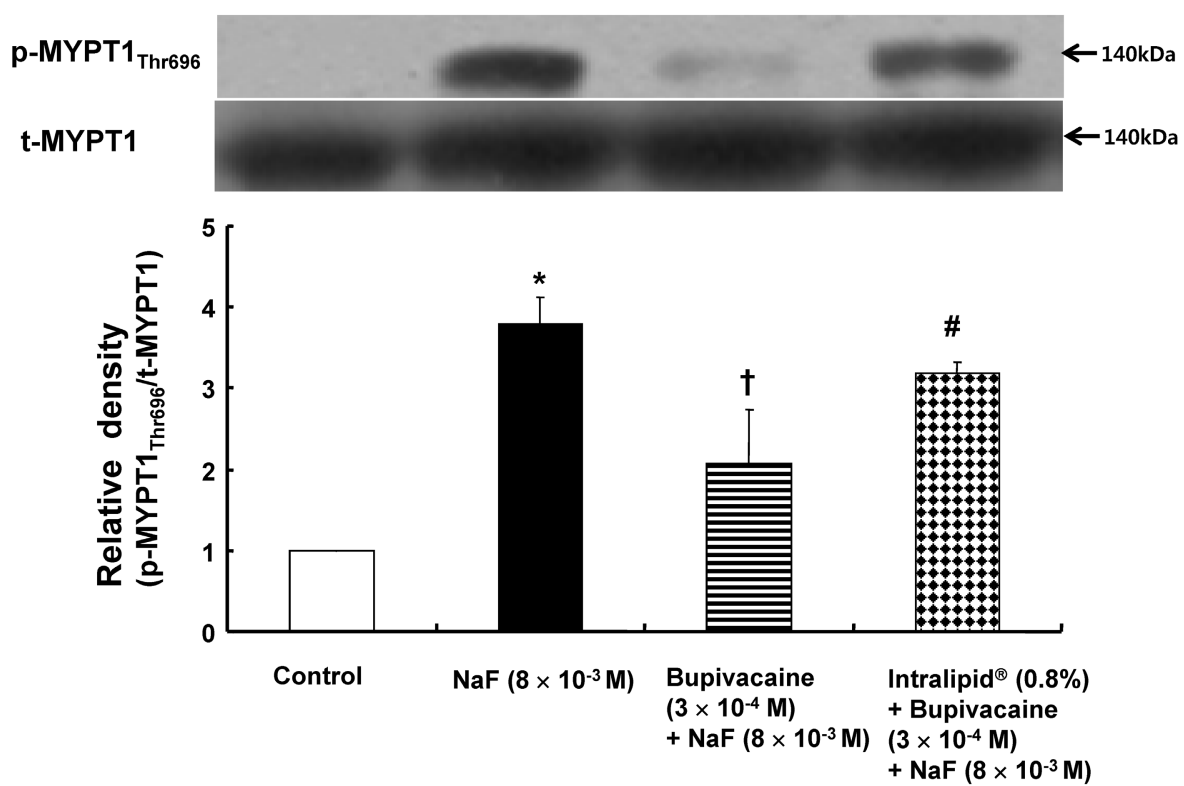

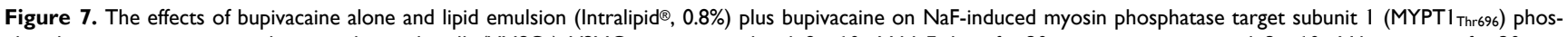
phorylation in rat aortic vascular smooth muscle cells (VMSCs). VSMCs were treated with $8 \times 10^{-3} \mathrm{M} \mathrm{NaF}$ alone for 30 min, pretreatment with $3 \times 10^{-4} \mathrm{M}$ bupivacaine for 30 min, followed by $8 \times 10^{-3} \mathrm{M} \mathrm{NaF}$ for $30 \mathrm{~min}$, and pretreatment with $0.8 \%$ lipid emulsion for $30 \mathrm{~min}$, followed by post-treatment with $3 \times 10^{-4} \mathrm{M}$ bupivacaine for 30 min and a subsequent 30-min treatment with $8 \times 10^{-3} \mathrm{M} \mathrm{NaF}$. Phosphorylation of MYPT1 on Thr696 was examined by western blot analysis as described in the methods. Data are expressed as the mean $\pm S D(n=3)$. $N$ indicates the number of independent experiments. $* P<0.001$ versus control. $\dagger P<0.01$ versus $8 \times 10^{-3} M N a F$ alone. $\# P<0.05$ versus $3 \times 10^{-4} M$ bupivacaine plus $8 \times 10^{-3}$ M NaF. P-MYPT1 Thr696: $_{\text {phosphorylated MYPT1 }}$ Thr696, t-MYPT1: total MYPT1.

Agonists including phenylephrine produce contraction mediated by calcium sensitization, which implies a higher increase in tension than in $\left[\mathrm{Ca}^{2+}\right]_{\mathrm{i}}$ [10]. Calcium sensitization mediated by PKC or Rho kinase inhibits MLCP via direct inhibition of MLCP or phosphorylation of MYPT1, leading to increased phosphorylation of regulatory light chain of myosin (MLC) and enhanced contraction [10]. The magnitude of bupivacaine-induced relaxation slightly differed in contracted aorta induced by either phenylephrine or $\mathrm{NaF}$ (Fig. 4). However, the magnitude of bupivacaine-induced relaxation was greatly lowered in PDBu-induced precontracted aorta than in phenylephrine-induced precontracted aorta (Fig. 4). In agreement with a previous report, Y-27632 attenuated contraction induced by phenylephrine and $\mathrm{NaF}$ (Fig. 3) [12]. Taken together, bupivacaine-induced vasodilation appears to be associated with the inhibition of contraction mediated by a pathway involving Rho kinase in isolated aorta precontracted with $\mathrm{NaF}$ or phenylephrine [12].

In agreement with a previous study, the lipid emulsion attenuated bupivacaine-induced vasodilation in aorta precontracted with phenylephrine but had no effect on mepivacaine-induced vasodilation [15]. It has been reported that lipid emulsions reverse vasodilation induced by toxic doses of bupivacaine and levobupivacaine, but have no effects on vasodilation induced by a toxic dose of mepivacaine, supporting the lipid sink theory that states that lipid emulsions extract high levels of highly lipid soluble local anesthetics from tissues [2-4]. Similar to previous reports, the lipid emulsion attenuated bupivacaine-induced vasodilation in aorta precontracted with $\mathrm{NaF}$, but had no effect on mepivacaine-induced vasodilation $[3,4,15]$. In addition, lipid emulsion pretreatment allows effective recovery from bupivacaine-induced cardiac toxicity in an isolated rat heart model, but not from mepivacaine-induced cardiac toxicity [16]. Post-treatment with lipid emulsions reduces heart rate recovery time from cardiac arrest induced by bupivacaine but not by mepivacaine [17]. These previous studies suggest that the potency of lipid emulsion-mediated scavenging effects of local anesthetics may be dependent on their lipid solubility (lipid/water partition coefficient: bupivacaine [27.5] versus mepivacaine [0.8]) [16-18]. Furthermore the lipid emulsion itself had no effect on contraction induced by $\mathrm{NaF}$ or phenylephrine, suggesting that the lipid emulsion has no effect on Rho kinase-mediated contraction induced by $\mathrm{NaF}$ or phenylephrine. Taking previous reports and current results into consideration, one of the putative mechanisms responsible for the lipid emulsion-mediated attenuation of bupivacaine-induced vasodilation can be explained as follows $[3,4,15-18]$. As the lipid solubility of bupivacaine is higher than that of mepivacaine, the lipid emulsion can extract higher amounts of bupivacaine than mepivacaine, leading to enhanced lipid emulsion-mediated attenuation of bupivacaine-induced 
vasodilation. In other words, the lipid emulsion-mediated attenuation of vasodilation induced by a toxic dose of bupivacaine appears to be associated with the relatively high lipid solubility of bupivacaine. However, lipid emulsion reverses the mepivacaine-induced blockade of the fast sodium current in cardiomyocytes [19]. This difference between previous and current studies may be ascribed to difference in the concentration of lipid emulsion ( 0.8 versus $10 \%)$, specimen (aorta versus cardiomyocyte) and experimental method (isometric tension measurement versus whole cell patch clamp). As contraction induced by phenylephrine and $\mathrm{NaF}$ was attenuated by $\mathrm{Y}-27632$ (Fig. 3), the lipid emulsion-mediated attenuation of vasodilation in precontracted aorta seems to be associated with the attenuation of the inhibitory effects of a toxic dose of bupivacaine on the Rho kinase-mediated pathway contributing to vascular smooth muscle contraction [12]. Bupivacaine-induced vasodilation in aorta precontracted with phenylephrine is mediated partially by decreased calcium sensitization [9]. Similar to results of a previous study, using aortic strips stimulated by $\mathrm{NaF}$, bupivacaine-induced vasodilation was enhanced compared with the bupivacaine-induced decrease in $\left[\mathrm{Ca}^{2+}\right]$ (Fig. 6), suggesting that decreased calcium sensitization partially contributes to the vasodilation via the inhibition of the Rho kinase-MYPT1 pathway induced by a toxic dose of bupivacaine $[9,10]$. As the lipid emulsion inhibited vasodilation induced by at toxic dose of verapamil (Fig. 5), the attenuation of vasodilation may be associated with the nonspecific inhibitory action of the lipid emulsion on bupivacaine-induced vasodilation [13].

In order to confirm that the lipid emulsion attenuates bupivacaine-induced vasodilation via the attenuation of the inhibition of the pathway involving Rho kinase and MYPT1, we examined the effects of bupivacaine alone and lipid emulsion plus bupivacaine on NaF-induced MYPT1 phosphorylation using western blotting. In agreement with a previous report, $\mathrm{NaF}$ induced MYPT1 phosphorylation in rat aortic VSMCs [12]. The phosphorylation of MYPT1 ${ }_{\text {Thr696 }}$ induced by Rho kinase inhibits MLCP, leading to the enhanced phosphorylation of MLC and increased contraction $[10,20]$. In the current study, a toxic dose $\left(3 \times 10^{-4} \mathrm{M}\right)$ of bupivacaine, which exceeds the approximate serum concentration $\left(10^{-5} \mathrm{M}\right)$ of bupivacaine that produces hypotension caused by bupivacaine-induced systemic toxicity, attenuated NaF-induced MYPT1 phosphorylation, whereas the lipid emulsion attenuated the bupivacaine-mediated inhibition of MYPT1 phosphorylation induced by NaF [21]. Taken together with previous reports and results from isometric tension measurements, the lipid emulsion-mediated attenuation of the inhibition of MYPT1 phosphorylation induced by a toxic dose of bupivacaine appears to contribute to the attenuation of vasodilation $[10,20]$. Further studies of the signaling pathway downstream of the lipid emulsion-mediated attenuation of the inhibition of the pathway involving Rho kinase and MYPT1 are needed to elucidate a detailed cellular signaling pathway.

The clinical relevance of the lipid emulsion-mediated attenuation of vasodilation induced by a toxic dose of bupivacaine should be carefully interpreted. The limitations of the current study are as follows: First, we used aorta, regarded as a conduit vessel, whereas small, resistance arterioles primarily contribute to the total peripheral vascular resistance, which is regarded as one of the most important determinants of blood pressure [22]. Second, we used endothelium-denuded aorta, whereas endothelium-derived vasodilators, including nitric oxide, endothelium-derived hyperpolarizing factor, and prostacyclin, modulate the vascular response [23]. Third, the lipid emulsion itself increases left ventricular systolic pressure via the blockade of nitric oxide release [11]. In addition, triglyceride microemulsion-induced reversal of bupivacaine toxicity is mediated by numerous factors, including scavenging and cardiotonic effects, fatty acid oxidation and drug redistribution $[24,25]$. Even with the above-mentioned factors that may modify the current results, the lipid emulsion may provide beneficial effects for recovery of vascular tone from vascular collapse induced by a toxic dose of bupivacaine.

In conclusion, these results suggest that lipid emulsions attenuate vasodilation induced by a toxic dose of bupivacaine via the attenuation of the inhibition of MYPT1 phosphorylation in isolated endothelium-denuded rat aorta. This effect appears to be associated with the high lipid solubility of bupivacaine.

\section{Abbreviation}

MYPT1: myosin phosphatase target subunit 1; PDBu: phorbol 12,13-dibutyrate; PKC: protein kinase C; MLC: regulatory light chain of myosin; MLCP: myosin light chain phosphatase; VSMC: vascular smooth muscle cell; $\left[\mathrm{Ca}^{2+}\right]_{\mathrm{i}}$ : intracellular calcium concentration.

\section{Acknowledgements}

This research was supported by the Basic Science Research Program through the National Research Foundation of Korea (NRF) funded by the Ministry of Education (2013R1A1A2057459). 


\section{Competing Interests}

The authors have declared that no competing interest exists.

\section{References}

1. Ozcan MS, Weinberg G. Update on the use of lipid emulsions in local anesthetic systemic toxicity: a focus on differential efficacy and lipid emulsion as part of advanced cardiac life support. Int Anesthesiol Clin 2011; 49:91-103.

2. Weinberg GL. Lipid emulsion infusion: resuscitation for local anesthetic and other drug overdose. Anesthesiology 2012; 117:180-7.

3. Ok SH, Sohn JT, Baik JS, Kim JG, Park SS, Sung HJ et al. Lipid emulsion reverses levobupivacaine-induced responses in isolated rat aortic vessels. Anesthesiology 2011; 114:293-301.

4. Ok SH, Han JY, Lee SH, Shin IW, Lee HK, Chung YK et al. Lipid emulsion-mediated reversal of toxic-dose aminoamide local anesthetic-induced vasodilation in isolated rat aorta. Korean J Anesthesiol 2013; 64:353-9.

5. Ok SH, Park CS, Kim HJ, Lee SH, Choi BH, Eun SY et al. Effect of two lipid emulsions on reversing high-dose levobupivacaine-induced reduced vasoconstriction in the rat aortas. Cardiovasc Toxicol 2013; 13:370-80.

6. Baik JS, Sohn JT, Ok SH, Kim JG, Sung HJ, Park SS et al. Levobupivacaine-induced contraction of isolated rat aorta is calcium dependent. Can J Physiol Pharmacol 2011; 89:467-76.

7. Choi YS, Jeong YS, Ok SH, Shin IW, Lee SH, Park JY et al. The direct effect of levobupivacaine in isolated rat aorta involves lipoxygenase pathway activation and endothelial nitric oxide release. Anesth Analg 2010; 110:341-9.

8. Sung HJ, Ok SH, Sohn JY, Son YH, Kim JK, Lee SH et al. Vasoconstriction potency induced by aminoamide local anesthetics correlates with lipid solubility. J Biomed Biotechnol 2012; Doi: 10.1155/2012/170958

9. Ok SH, Bae SI, Kwon SC, Park JC, Kim WC, Park KE et al Bupivacaine-induced vasodilation is mediated by decreased calcium sensitization in isolated endothelium-denuded rat aortas precontracted with phenylephrine. Korean J Pain 2014; 27:229-38.

10. Akata T. Cellular and molecular mechanisms regulating vascular tone. Part 2: regulatory mechanisms modulating $\mathrm{Ca}^{2+}$ mobilization and/or myofilament $\mathrm{Ca}^{2+}$ sensitivity in vascular smooth muscle cells. J Anesth 2007; 21:232-42.

11. Shin IW, Hah YS, Kim C, Park J, Shin H, Park KE et al. Systemic blockage of nitric oxide synthase by L-NAME increases left ventricular systolic pressure, which is not augmented further by Intralipid ${ }^{\circledR}$. Int J Biol Sci 2014; 10:367-76.

12. Jeon SB, Jin F, Kim JI, Kim SH, Suk K, Chae SC et al. A role for Rho kinase in vascular contraction evoked by sodium fluoride. Biochem Biophys Res Commun 2006; 343:27-33.

13. Pang DC, Sperelakis N. Uptake of calcium antagonistic drugs into muscles as related to their lipid solubilities. Biochem Pharmacol 1984; 33:821-6.

14. Shim HS, Ok SH, Lee SH, Kwon SC, Sohn JT. Protein kinases participate in the contraction in response to levobupivacaine in the rat aorta. Eur J Pharmacol 2012; 677:131-7.

15. Lee SH, Sung HJ, Ok SH, Yu J, Choi MJ, Lim JS et al. 2013. Lipid emulsions enhance the norepinephrine-mediated reversal of local anesthetic-induced vasodilation at toxic doses. Yonsei Med J 2013; 54:1524-32.

16. Aumeier C, Kasdorf B, Gruber M, Busse H, Wiese CH, Zink W et al. Lipid emulsion pretreatment has different effects on mepivacaine and bupivacaine cardiac toxicity in an isolated rat heart model. Br J Anaesth 2014; 112:735-41.

17. Zausig YA, Zink W, Keil M, Sinner B, Barwing J, Wiese $\mathrm{CH}$ et al. Lipid emulsion improves recovery from bupivacaine-induced cardiac arrest, but not from ropivacaine- or mepivacaine-induced cardiac arrest. Anesth Analg 2009; 109:1323-6.

18. Heavner JE. Local anesthetics. Curr Opin Anaesthesiol 2007; 20:336-42

19. Wagner M, Zausig YA, Ruf S, Rudakova E, Gruber M, Graf BM, et al. Lipid rescue reverses the bupivacaine-induced block of the fast $\mathrm{Na}+$ current (INa) in cardiomyocytes of the rat left ventricle. Anesthesiology. 2014; 120:724-36.

20. Feng J, Ito M, Ichikawa K, Isaka N, Nishikawa M, Hartshorne DJ et al. Inhibitory phosphorylation site for Rho-associated kinase on smooth muscle myosin phosphatase. J Biol Chem 1999; 274:37385-90

21. Santos AC, DeArmas PI. Systemic toxicity of levobupivacaine, bupivacaine, and ropivacaine during continuous intravenous infusion to nonpregnant and pregnant ewes. Anesthesiology 2001; 95:1256-64.

22. Christensen KL, Mulvany MJ. Location of resistance arteries. J Vasc Res 2001; 38:1-12.

23. Busse R, Fleming I, Hecker M. Signal transduction in endothelium-dependent vasodilatation. Eur Heart J 1993; 14 (Suppl I):2-9.

24. Fettiplace MR, Lis K, Ripper R, Kowal K, Pichurko A, Vitello D et al. Multi-modal contributions to detoxification of acute pharmacotoxicity by a triglyceride micro-emulsion. J Control Release 2015; 198C:62-70

25. Partownavid P, Umar S, Li J, Rahman S, Eghbali M. Fatty-acid oxidation and calcium homeostasis are involved in the rescue of bupivacaine-induced cardiotoxicity by lipid emulsion in rats. Crit Care Med 2012; 40:2431-7. 